\title{
Embedded-Circuit Magnetic Metamaterial Substrate Performance for Patch Antennas
}

Kevin Buell*, Hossein Mosallaei and Kamal Sarabandi

Radiation Laboratory at the Department of Electrical Engineering and Computer Science University of Michigan

Ann Arbor, MI 48109-2122, USA

E-mail: kbuell@umich.edu, hosseinm@umich.edu, saraband@eecs.umich.edu

Abstract

Magnetic permeability was imparted to a naturally non-magnetic material by metallic inclusions. A patch antenna tested the performance of the magnetic metamaterial as a substrate. The magnetic metamaterial exhibited enhanced $\mu$ and $\varepsilon$ as predicted with acceptable loss-factor levels. Models for predicting $\mu$ and $\varepsilon$ are presented and simulations indicate that permeability can be extended well beyond several gigahertz with current technology. miniaturization factor=4-6.4 with efficiency $=21-35 \%$

A. Background and Goals

I. INTRODUCTION

$7^{\text {OR low loss applications in the microwave region natural material choices are limited to non-magnetic }}$ 1 dielectrics. The gyromagnetic resonances limits the upper frequency end of the magnetic region in natural materials occur in the VHF-UHF range, too low a frequency for microwave applications. To our knowledge there is no material available with moderately low loss $\left(\operatorname{Tan} \delta_{m}=\frac{\mu^{\prime \prime}}{\mu^{2}}<0.02\right)$ and moderately enhanced permeability $(\mu>2)$ for operation deeply into the microwave region,

I) Benefits of Magnetic Materials: The permittivity of a composite dielectric can be engineered for
enhanced permeability $(\mu>2)$ for operation deeply into the microwave region, I) Benefits of Magnetic Materials: The permittivity of a composite dielectric can be engineered for
practically any permittivity desirable. In contrast, in the microwave region the permeability of low loss materials is limited to that of free space.

High dielectric constant materials are used to achieve electromagnetic scaling and field confinement. Use of high dielectrics is limited by the dramatic mismatch in wave impedance relative to feed networks and free space. But if permeability can be increased from that of free-space, the $\mu, \varepsilon$ product increases quickly with relative permeability, providing miniaturization and electromagnetic scaling $\left(\lambda_{\text {medium }}=\frac{\lambda_{0}}{\mu_{e s}}\right)$. As relative permeability increases to match relative permittivity the $\mu, \varepsilon$ ratio and the intrinsic impedance of the medium $\left(\eta=\sqrt{\frac{\mu_{r}, \psi_{0}}{\varepsilon_{1} \varepsilon_{0}}}\right)$ can be driven to match that of free space. This decreases EM field confinement in magnetodielectric materials.

The benefit to minimizing the energy lost due to the reflection at this interface is obvious and further anticipated benefits are improvements in matching and bandwidth.

Consider the case of a patch antenna over a dielectric and ground-plane. Simulations show that for an aggressive miniaturization with $\varepsilon_{r}=25, \tan _{\delta e}=0.001$ field confinement limits bandwidth $(0.64 \%)$ and efficiency $(77 \%)$. With magnetic materials, $\varepsilon_{r}=\mu_{r}=5, \tan _{\bar{\delta}}=\tan _{\delta m}=0.001$ field confinement is alleviated efficiency $\left(77 \%\right.$. With magnetic materials, $\varepsilon_{r}=\mu_{r}=5, \tan _{\tilde{\delta}_{e}}=\tan _{\delta m}=0.001$ field confinement is alleviated
and bandwidth improves by a factor of better than ten $(7.94 \%)$ and efficiency reaches an almost perfect value and band of $(99 \%)$.

B. Embedded Circuit Meta-Material

The project for this paper is to achieve enhanced positive magnetic permeability and electric permittivity for low loss microwave applications. Recently, geometries optimized to provide superior magnetic properties have been considered theoretically [6], but to our knowledge none have been proven useful in practical experiment.

Our circuit geometry is engineered to control energy coupling and storage. The benefit of this control is that within the limits of the processing technology the effective permittivity and most importantly effective permeability of the medium can be tailored to the demands of the application.

$$
\text { II. EFFective Medium Operation }
$$

Magnetic energy storage is the definitive characteristic of permeable materials. When magnetic energy storage is achieved by artificial means an effective permeability is observed. The basic circuit unit for magnetic energy storage is the inductor and an electromagnetically small inductor embedded into a dielectric material will store any coupled magnetic energy similarly to the manner in which magnetic energy is stored in the electron motion of naturaliy magnetic materials.

Figure 1 shows an embedded circuit capable of producing magnetic properties in a natural dielectric. The spiral loop acts as an inductor, coupling energy from an incident magnetic field to produce a current loop in the spiral. There is a distributed capacitance between the loops of the spiral, and the interaction between the 
spiral inductance and spiral capacitance causes the resonant behavior. Near resonance the current magnitude in the spiral loop increases and the magnetic permeability is enhanced.

To achieve an effective medium behavior the embedded circuits must be distributed uniformly through the host dielectric. Planar microstrip processing is employed and the resulting substrate-metallization layers are stacked to form an effective medium. This method of assembly allows for the critical control of geometrically stacked to form an effective medium. This method of assembly allows for the critical control of
determined circuit parameters and thereby selection of resonant frequency and coupling factors.

\section{A. Equivalent Circuit Mode}

The spiral loop of Figure 1 may be modelled as a simple resonator (Fig. 2. Incorporating the spiral loop loss mechanisms, the equivalent circuit model for the composite medium is given in Figure 3.

1) Capacitance: To model the equivalent lumped-element capacitance $\left(\mathcal{C}_{\text {spiral }}\right.$ in figure 2$)$, the primary capacitive effect to be considered is the capacitance between adjacent wraps of the spiral inductor. Additional smaller capacitances will result from the interaction between non-adjacent wraps, but only considering the adjacent wraps gives an understanding of the physics involved.

The distributed capacitance of the spiral inductor can be determined by considering the geometry of the "capacitive spiral" indicated in Fig. 1 by a dotted line which traces the path between the metallic spiral arms. The basic form of the capacitive equation (1) is the capacitance per unit length of co-planar thin arms. The basic form of the capacitive equation (1) is the capacitance per unit length of co-planar thin
metallic strips multiplied by the length of the strips and the dielectric constant of the host medium. An metallic strips multiplied by the length of the strips and the dielectric constant of the host medium. An
elliptical integral (3) exists for determining the capacitance per unit length, and (5) gives the spiral length. In equations $1-5$ the width of the trace metallization is indicated by ' $W$ ' and the inter-trace gap spacing is ' $\mathrm{S}$ '. In (5), $\mathrm{N}$ is the number of turns of the metallic spiral arm and $\mathrm{n}=\mathrm{N}-1$ is the number of turns of the capacitive spiral gap. Eq. (5) is correct for integer or half-integer values of $\mathrm{N}$, and integer/4 values if $\mathrm{Ll}=\mathrm{L} 2$.

2) Inductance: The planar elements in Figure 1 are stacked along the Y-dimension with a spacing of $\Delta Y$. This geometry effectively forms a solenoid along the $Y$-axis of spiral loop elements and because of the long-solenoid structure, a uniform field distribution can be assumed. Eq. (2) is the inductance for a lons thin solenoid of length "len", "N" turns, and a cross-sectional area "S". The inductance of a single spira loop element in this solenoid is just slightly lower than that given by the solenoid inductance equation if the 'fill-ratio' is low ( $\mathrm{N}$ is small).

3) Resonance: Once the distributed capacitance and inductance of the spiral loop are known from the methods above, the resonance frequency of the embedded circuit can be estimated $F_{\text {res }}=\frac{1}{\sqrt{u_{\text {m }}} \text {. This }}$ estimate of resonance frequency is generally high. Some significant capacitances and inductances are being neglected by the analytical method and more rigorous numerical simulation methods should also be employed To more accurately model the medium, a finite element solver such as the commercially available HFSS is To more accurately model the medium, a finite element solver such as the commercially available HFSS is
helpful. Perfect Electrically Conducting (PEC) walls and Perfectly Magnetically Conducting (PMC) boundary conditions around a single element unit can be employed to enforce symmetry conclitions and emulate an infinite YZ plane of embedded circuits as shown in Figure 4.

4) Effective Medium: In order to form an effective medium as represented by this model, a planar array of the unit-cell of figure 1 is printed onto an $\mathrm{XZ}$ planar surface. These infinite $\mathrm{XZ}$ grid planes may be stacked infinitely in the $Y$ dimension to form a 3D infinite medium.

Analytic formulations for the effective bulk permittivity and permeability of such embedded circuit metamaterials exist which correlate to the geometry of Fig. 3. [6]

5) Permeability: The effective-medium design provides permeability enhancement only along the solenoida 5) Permeability: The effective-medium desigg
axis. The permeability tensor is given in (10).

Effective permeability $\left(\mu_{e f f}\right)$ is given in equation 6 and is a function of the resonant frequency of the spiral inductors $\omega_{p}$, the frequency of the incident field $\omega$, the resonator quality factor ' $Q$ ' and the coupling coefficient of Y-directed magnetic energy $\kappa$. Figure 6 shows a typical response of $\mu_{e f f}$ to frequency variation. Operating values of $\mu_{r}=1-4$ can be achieved with moderately low-loss performance.

The coupling coefficient $K$ and most other metamaterial properties are a function of the circuit geometry shown in Figure 1. The resonator ' $Q$ ' in $(9)$ is a function of the metal conductivity $\sigma$ Care should be taken to observe the condition that conductor thickness $\tau>2 \delta$, where $\delta=\sqrt{\frac{2}{\alpha \alpha_{0} \sigma \sigma}}$ is the metallization skin depth at the frequency of operation.

6) Permittivity: Consider an X-directed electric ficld. Along the majority of the X-dimension, the electric field is shorted by the metallization of embedded circuit loop parallel to the incident $\mathrm{E}$-field. In the gap-region between the unit-cells, the Z-directed metallizations form inter-cell capacitors for the incident X-directed Electric field. This capacitance is what stores electrical energy and provides for the X-directed permirtivity of (7). The same phenomenon is observed in the Z-Dimension, but in the Y-dimension $\left(\varepsilon_{r}=\varepsilon_{\text {diel }}\right)$. The corresponding permittivity tensor is given in Equation 7.

Unlike the permeability, the effective permittivity of the medium is not frequency dependent in the microwave region. The permittivity in (7) is a function of the inter-cell capacitance which is calculated 
by means of the same elliptical integral that was used to find the spiral capacitance (Equation 3). This analysis assumes a medium where the circuits are embedded in a 'simple' dielectric with $\mu_{r}=1$.

Figure 5 demonstrates the proper orientation for a patch antenna operating in the regular mode to benefit from both $\mu_{e f f}$ and $\varepsilon_{e f f}$. In the area under the patch, the image reflections from the metallic antenna and ground plane appear to form an infinite medium in the Z-dimension, allowing the effective medium analysis ground plane appear to form an infinite medium in the $Z$-dies.
above to approximately apply despite the finite geometries.

III. Metamaterial Design and Fabrication

The metamaterial substrate was designed with a cell-size of $\Delta x=\Delta z=2 \mathrm{~cm}, \Delta y=3.028 \mathrm{~mm}(120 \mathrm{mils})$ The substrate was fabricated on 120 mil thick Rogers RO-4003 dielectric. The spiral resonators were etched from $1 / 2$ oz thick copper $(0.017 \mathrm{~mm})$ with a line-width (w) and spacing (s) of $0.127 \mathrm{~mm}(5 \mathrm{mils})$. For our design $l x=l z=16 \mathrm{~mm} . W=S=0.127 \mathrm{~mm}$ and Length $W$ piralGap $=6.24 \mathrm{~mm}$. Then for a Rogers RO-4003 design $l x=l z=16 \mathrm{~mm} . W=S=0.127 \mathrm{~mm}$ and $L$ ength SpiralGap $=6.24 \mathrm{~mm}$. Then for a Rogers RO-4003
dielectric host medium $\varepsilon_{r}=3.38$ and $C_{S \text { piral }}=5.3 \mathrm{pF}$. len $=\Delta Y=3.028 \mathrm{~mm}, N=2$, and $S \approx 2.56 \times 10^{-4} \mathrm{M}^{2}$

dielectric host medium $\varepsilon_{r}=3$.
and $L_{\text {spiral }} \approx L=.422 \mu H$.

To reduce substrate mass $33 / 64$ 'th inch diameter air-holes were drilled along the y-axis into the center of each spiral resonator cell. The final substrate mass is reduced by a factor of approximately $1 / 3$ 'rd which is significant for a $2 \mathrm{~cm}$ thick substrate.

The final substrate was formed by stacking XZ-planar strips in the Y-dimension to form the final substrate in the geometry of figure 5. The final substrate was 24 cells in the $x$-dimension $x 75$ cells in the $y$-dimension $x$ one cell in the z-dimension. The resulting total substrate geometry was $24 \mathrm{~cm} \times 24 \mathrm{~cm} \times 2 \mathrm{~cm}$ and had a weight of about 3.5 pounds.

IV. REsults

A. Metamaterial Characteristic

The metamaterial resonance frequency was measured to be $285 \mathrm{MHz}$. This is a reasonable match to the resonance predicted by numerical simulation in the FEM method, which was $250 \mathrm{MHz}$. The $\varepsilon_{r(m e t a)}$ and $\mu_{r(\text { meta })}$ for this design are predicted in Fig. 6. Equations (7) and (6) predict that at $250 \mathrm{MHz} \varepsilon_{r(m e t a)}=9.6$, $\mu_{r(m e t a)}=3.1$, and $\operatorname{Tan} \delta_{m} 0.0073$.

B. Antenna Performance

A probe-fed microstrip patch antenna resonant at $250 \mathrm{MHz}$ was built on the metamaterial substrate. To resonate at $250 \mathrm{MHz}$ the patch dimensions were $9.3 \times 9.3 \mathrm{~cm}$.

The $250 \mathrm{MHz}$ antenna performance parameters are given in Table I and Table II compares the miniaturization and efficiency factors of several antenna geometries operating at different frequencies.

To provide comparison, the antenna probe feed position and geometry were also adjusted to provide a resonant dimension along the $\mathrm{Y}$-axis rather than the $\mathrm{X}$-axis. In this orientation there should be no effective permeability experienced by the antenna, but permittivity should remain enhanced. That is $\mu_{r}=1$ but $\varepsilon_{r z}$ is given by equation 7 .

The orientation and frequency dependence of miniaturization factor reinforces validation of the embedded spiral loop resonator model.

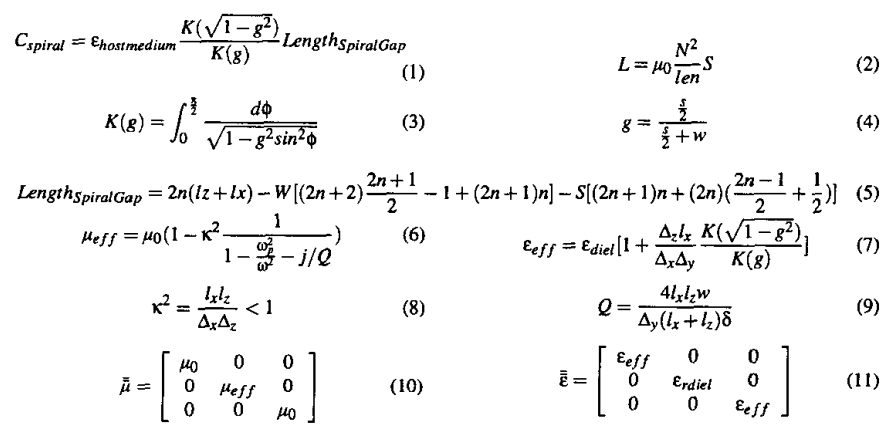




\begin{tabular}{|c|c|}
\hline \multicolumn{2}{|c|}{$250 \mathrm{MHz}$ Patch Antenna } \\
over Magnetic Metarnaterial Substrate \\
\hline Insertion Loss & $-11.9 \mathrm{~dB}$ \\
-10dB IL BW & $0.83 \%$ \\
Gain & $-3.9 \mathrm{dBi}$ \\
Directivity & $3.03 \mathrm{dBi}$ \\
Cross-Polarization & $-12 \mathrm{~dB}$ \\
Efficiency & $21.6 \%$ \\
Miniaturization Factor & 6.4 \\
\hline \multicolumn{2}{|c}{ TABLE I }
\end{tabular}

AnTenna Parameters

\begin{tabular}{|c|c|c|c|}
\hline \multicolumn{4}{|c|}{ Miniaturization and Efficiency } \\
\hline Description & Frequency & $\begin{array}{c}\text { Miniaburization } \\
\text { Factor }\end{array}$ & Efficiency \\
\hline Anti-Aligned & $250 \mathrm{MHZ}$ & 3.1 & $12 \%$ \\
Aligned & $235 \mathrm{MHZ}$ & 4.0 & $35.2 \%$ \\
Aligned & $240 \mathrm{MHZ}$ & 5.0 & \\
Aligned & $245 \mathrm{MHZ}$ & 5.3 & \\
Aligned & $250 \mathrm{MHZ}$ & 6.4 & $21.6 \%$ \\
\hline \multicolumn{4}{|c}{ TABLE II }
\end{tabular}

EFFCIENCY AND MINIATURIZATION

\section{CONCLUSION}

A naturally non-magnetic dielectric was imbued with magnetic properties and permeability by means of embedded resonant circuits. This metamaterial substrate possesses enhanced positive values of permeability with reasonable loss levels. A microstrip patch antenna was developed and tested to demonstrate the potential application of these embedded circuit magnetic metamaterial substrates. This experiment indicates validity of the metamaterial analytical model. It is predicted that a wide range of applications will benefit from moderate or low-loss magnetic metamaterials in the microwave region.

Simulations indicate that with commercially available materials and processing technology, the production of effective permeability magnetic metamaterials by this technique can be easily achieved in frequencies of several gigahertz for permeability in the $\mu_{r}=1-4$ range for moderately low-loss applications.
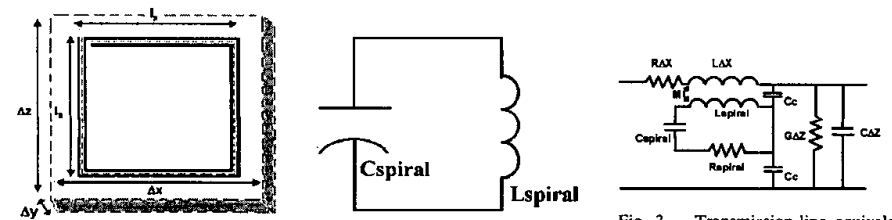

Fig. 3. Transmission-line equivalent

Fig. 1. The metamaterial unit cell. $\Delta_{x}$. Fig. 2 . The spiral loop equivalent model for magnetic metamaterial. $\Delta_{y}$, and $\Delta_{z}$ is the unit cell size. $N=2$ lumped-element circuit model.

is the number of wraps of the spiral.

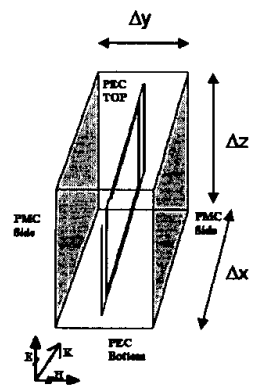

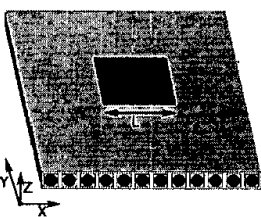

Fig. 5. Patch antenna over Magnetic Metamaterial Substrate. Length ' $\mathrm{L}$ ' is the resonant length and indicates orientation of radiating current.

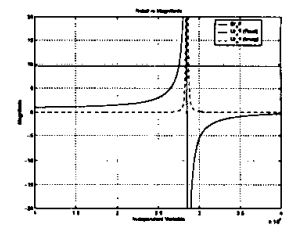

Fig. 6. Relative Permittivity, Permeability, and Magnetic Loss Tangent. At $250 \mathrm{MHz} \varepsilon_{r(\text { meta })}=9.6, \mu_{\text {rimeta })}=3.1$,
and $\operatorname{Tan}_{m} 0.0073$.

Fig. 4. FEM Boundary condition

to test resonance frequency.

REFERENCES

[1] N. Engheta. MMET '02. 2002 International Conference. vol. 1 pp. 175-180, 10-13 Sept. 2002

[2] N. Engheta, IEEE MTT-S IntemationalMicrowave Symposilm Digest, 2003. vol. 1, pp. 187-190, 8-13 June 2003

[3] R.W. Ziolkowski,IEEE Ant. and Prop. Soc. Intemational Symposium, 2002., vol: 2, pp. 396-399, 16-21 June 2002

[4] R.W. Ziolkowski, IEEE Trans. on Antennas and Propagation, vol. 51 iss. 7, pp. $1516-1529$ July 2003

5] D.J. Kem, D.H. Werner, Ant. and Prop., 2003 IEEE Soc. Intermat. Conf. Vol: 1, Jun 22-27, 2003 pp: $497-50030$

6] K. Sarabandi, H. Mosallaei, "Electro-Ferromagnetic Tunable Permeability, Band-Gap, and Bi-Anisotropic Meta-

Materials Utilizing Embedded-Circuits," IEEE AP-S International Symposium, Columbus, Ohio, June 22-27, 2003.

[7] K.Buell, K. Sarabandi "Measurement of Meta-Materials Utilizing Resonant Embedded-Circuit For Artificial Permeability By Frequency Extended Perturbation Method" IEEE AP-S International Symposium, Columbus, Ohio, June $22-27,2003$ 